\title{
Who would really benefit from DPP-4 inhibitors?
}

\author{
Nasser Mikhail
}

Received: 15 January 2014/ Accepted: 25 January 2014/Published online: 8 February 2014

(C) Springer Science+Business Media New York 2014

Since the introduction of DPP-4 inhibitors in 2006, this class of drugs has increasingly become a strong competitor of sulfonylureas (SU) as second-line therapy in patients with type 2 diabetes uncontrolled on metformin. The use of DPP-4 inhibitors is associated with neutral effect on body weight and low risk of hypoglycemia except when used in conjunction with SU or insulin. These two advantages make DPP-4 inhibitors as convenient therapeutic option in patients who are overweight and subjects susceptible for hypoglycemia. The third advantage is the possibility of using these agents in diabetic patients with chronic kidney disease and those on hemodialysis [1]. They can be used, therefore, as alternative to metformin in patients with various stages of kidney disease.

However, it is still unclear whether there exist patients' clinical and biochemical characteristics that may influence the efficacy of DPP-4 inhibitors. In this issue of Endocrine, Esposito et al. [2] performed a meta-regression analysis of 20,053 patients exposed to various DPP-4 inhibitors in 78 randomized trials that lasted 12 weeks or longer. The authors found that higher values of $\mathrm{HbAlc}$ and lower fasting hyperglycemia at baseline were the most two important predictors of greater reductions in $\mathrm{HbAlc}$ values, whereas age did not modify the efficacy of DPP-4 inhibitors [2].

The analysis of Esposito et al. [2] included the largest available database used to study determinants of efficacy of all available DPP-4 inhibitors. Clearly, the results of this metaanalysis represent a step forward for better understanding of

\section{N. Mikhail $(\square)$}

Endocrinology Division, Olive-View UCLA Medical Center,

David-Geffen School of Medicine, 14445 Olive View Dr,

Sylmar, CA 91342, USA

e-mail: nmikhail@dhs.lacounty.gov this area of medicine characterized by scanty and sometimes inconsistent investigations. However, the study had some limitations besides those already mentioned by the authors. First, no attempts were undertaken to include unpublished studies to minimize publication bias. Second, the exclusion of 11 trials of initial combination of DPP-4 inhibitors with other oral agents and insulin may not be necessary because the use of these combinations in clinical practice is not uncommon, and their inclusion would have increased the study's statistical power. Surprisingly, the authors did not mention an earlier meta-analysis designed for the same purpose by the group of Manomi and co-workers [3] including 44 trials of 21-week duration or longer. In the latter study, the authors also reported a greater efficacy of DPP-4 inhibitors in patients with lower fasting plasma glucose concentrations [3]. Yet, unlike the study of Esposito and co-workers [2], Monami et al. [3] found that older age and unexpectedly lower HbA1c values at baseline predicted a greater therapeutic response to DPP-4 inhibitors. Moreover, diabetes duration, an important clinical parameter that was not analyzed as covariate in the study of Esposito et al. [2], did not affect the efficacy of DPP-4 inhibitors in the meta-analysis performed by Monami and his colleagues [3].

Other investigators explored several possible determinants of DPP-4 efficacy. In one nonrandomized study $(n=109)$, Fadini et al. [4] observed that the presence of the metabolic syndrome, irrespective of its definition, predicted lesser $\mathrm{HbA} 1 \mathrm{c}$ reduction in response to sitagliptin and vildagliptin. In the same study, neither age nor diabetes duration modified the efficacy of these two DPP-4 inhibitors [4]. Biochemical markers such as plasma DPP-4 activity may virtually serve as predictor of efficacy of DPP-4 inhibitors. In another nonrandomized study $(n=52)$, Aso et al. [5] found that high serum levels of soluble CD26 (sCD26) were associated with poor efficacy 
to sitagliptin used in submaximal doses of $50 \mathrm{mg} / \mathrm{d}$ (the standard dose in Japan). Indeed, there is strong correlation between serum levels of sCD26 and DPP-4 activity suggesting that serum sCD26 may reflect DPP-4 activity [5]. Another approach to examine various predictors of therapeutic response to DPP-4 inhibitors may be achieved by analysis of predefined patient subgroups involved in large randomized trials. In this respect, two recent trials that evaluated safety of saxagliptin and alogliptin in diabetic patients with high cardiac risk did not report any particular patient subgroup that responds differently to these two agents in terms of $\mathrm{HbAlc}$ reduction [6, 7]. Unfortunately, despite a modest decrease in HbA1c values, averaging $0.2 \%$ with saxagliptin and $0.36 \%$ with alogliptin compared with placebo, there was no cardiovascular or mortality benefit demonstrated over a median duration of follow-up of 2.1 and 1.5 years in the saxagliptin trial and alogliptin trial, respectively [6, 7]. On the contrary, in the saxagliptin study, there was significant increase in proportions of patients hospitalized for heart failure. These proportions were 3.5 and $2.8 \%$ of subjects randomized to saxagliptin and placebo, respectively, yielding a hazard ratio of 1.27 (95\% CI 1.07-1.51, $P=0.007$ ). Although it is premature to conclude that saxagliptin will replicate the rosiglitazone story, it is prudent not to simply focus on the extent of HbA1c reduction by DPP-4 inhibitors or other antidiabetic agents. Rather, our objective should be to derive the knowledge of whether amelioration of glycemic control by a given drug would lead to decrease in cardiovascular events and mortality in all patients or in specific subgroups. Achievement of such goal will allow us to identify the patients who would really benefit from DPP-4 inhibitors.

Conflicts of interest The author has no conflict of interest to disclose.

\section{References}

1. N. Mikhail, Use of dipeptidyl peptidase-4 inhibitors for the treatment of patients with type 2 diabetes and chronic kidney disease. Postgrad. Med. 124, 138-144 (2013)

2. K. Esposito, P. Chiodini, A. Capuano, M.I. Maiorino, G. Bellastella, D. Giugliano, Baseline glycemic parameters predict the hemoglobin A1c response to DPP-4 inhibitors. Endocrine (2013). doi:10.1007/s12020-013-0090-0

3. M. Monami, F. Cremasco, C. Lamanna, N. Marchionni, E. Mannucci, Predictors of response to peptidyl peptidase- 4 inhibitors: evidence from randomized trials. Diab. Metabol. Res. Rev. 27, 362-372 (2011)

4. G.P. Fadini, S.V. de Kreutzenberg, R. Gjini, A. Avogare, The metabolic syndrome influences the response to incretin-based therapies. Acta Diabetol. 48, 219-225 (2011)

5. Y. Aso, N. Ozeki, T. Terasawa, R. Naruse, K. Hara, M. Suetsugu, K. Takebayashi, M. Shibazaki, K. Haruki, K. Morita, T. Inukai, Serum levels of soluble CD26/dipeptidyl peptidase-4 (DPP-4) predicts the response to sitagliptin, a DPP-4 inhibitor, in patients with type 2 diabetes controlled inadequately by metformin and/or sulfonylurea. Transl. Res. 159, 25-31 (2012)

6. B.M. Scirica, D.L. Bhatt, E. Braunwald, et al., for the SAVORTIMI 53 Steering Committee and Investigators, Saxagliptin and cardiovascular outcomes in patients with type 2 diabetes mellitus. N. Engl. J. Med. 369, 1317-1326 (2013)

7. W.B. White, C.P. Cannon, S.R. Heller, et al., for the EXAMINE Investigators, Alogliptin after acute coronary syndrome in patients with type 2 diabetes. N. Engl. J. Med. 369, 1327-1335 (2013) 